Fixed Point Theory, 22(2021), No. 2, 933-946

DOI: $10.24193 /$ fpt-ro.2021.2.61

http://www.math.ubbcluj.ro/ nodeacj/sfptcj.html

\title{
POSITIVE SOLUTIONS OF NONLINEAR THIRD-ORDER BOUNDARY VALUE PROBLEMS INVOLVING STIELTJES INTEGRAL CONDITIONS
}

\author{
LIU YANG, HUI ZHOU AND CHUNFANG SHEN
}

College of Mathematics and Statistics, Hefei Normal University, Hefei, 230061, PR China

\begin{abstract}
In this paper, by using the Guo-Krasnoselskii theorem, we investigate the existence and nonexistence of positive solutions of a class of boundary value problem of third-order nonlinear differential equation involving Stieltjes integral conditions. Under some growth conditions imposed on the nonlinear term, we obtain explicit ranges of values of parameters with which the problem has a positive solution and has no positive solution respectively. An example is given to illustrate the main results of the paper.
\end{abstract}

Key Words and Phrases: Positive solution, boundary value problem, fixed point, cone.

2020 Mathematics Subject Classification: 45C05, 34B18, 47H10.

Acknowledgement. The work is sponsored by the Natural Science Foundation of China (12001152), Anhui Provincial Natural Science Foundation (2008085AQ08), the Higher School Natural Science Project of Anhui Province (KJ2020A0089, KJ2019A0712, gxyq2019067).

\section{REFERENCES}

[1] D.R. Anderson, Multiple positive solutions for a three-point boundary value problem, Math. Comput. Modelling, 27(6)(1998), 49-57.

[2] D.R. Anderson, Green's function for a third-order generalized right focal problem, J. Math. Anal. Appl., 288(2003), 1-14.

[3] J. Chu, Z. Zhou, Positive solutions for singular non-linear third-order periodic boundary value problems, Nonlinear Anal., 64(2006), 1528-1542.

[4] M. El-Shahed, Positive solutions for nonlinear singular third order boundary value problem, Commmun. Nonlinear Sci. Numer. Simlat., 16(2011), 402-413.

[5] J.R. Graef, L. Kong, Positive solutions for third order semipositone boundary value problems, Appl. Math. Lett., 22(2009), 1154-1160.

[6] J. Graef, J.R.L. Webb, Third order boundary value problems with nonlocal boundary conditions, Nonlinear Anal., 71(2009), 1542-1551.

[7] M. Gregus, Third Order Linear Differential Equations, in: Math. Appl., Reidel, Dordrecht, 1987.

[8] D. Guo, V. Lakshmikantham, Nonlinear Problems in Abstract Cones, Academic Press, New York, 1988. 
[9] L. Guo, J. Sun, Y. Zhao, Existence of positive solutions for nonlinear third-order three-point boundary value problems, Nonlinear Anal., 68(2008), 3151-3158.

[10] B. Hopkins, N. Kosmatov, Third-order boundary value problems with sign-changing solutions, Nonlinear Anal., 67(2007), 126-137.

[11] G. Infante, J.R.L. Webb, Loss of positivity in a nonlinear scalar heat equation, NoDEA Nonlinear Differential Equations Appl., 13(2006), 249-261.

[12] T. Jankowski, Existence of positive solutions to third order differential equations with advanced arguments and nonlocal boundary conditions, Nonlinear Anal., 75(2012), 913-923.

[13] R.W. Leggett, L.R. Williams, Multiple positive fixed points of nonlinear operators on ordered Banach spaces, Indiana Univ. Math. J., 28(1979), 673-688.

[14] S. Li, Positive solutions of nonlinear singular third-order two-point boundary value problem, J. Math. Anal. Appl., 323(2006), 413-425.

[15] X. Lin, Z. Du, W. Liu, Uniqueness and existence results for a third-order nonlinear multi-point boundary value problem, Appl. Math. Comput., 205(2008), 187-196.

[16] P. Minghe, S.K. Chang, Existence and uniqueness of solutions for third-order nonlinear boundary value problems, J. Math. Anal. Appl., 327(2007), 23-35.

[17] A.P. Palamides, G. Smyrlis, Positive solutions to a singular third-order three-point boundary value problem with an indefinitely signed Green's function, Nonlinear Anal., 68(2008), 21042118.

[18] J. Webb, G. Infante, Positive solutions of nonlocal boundary value problems: a unified approach, J. London Math. Soc., 74(2006), no. 2, 673-693.

[19] J. Webb, K. Lan, Eigenvalue criteria for existence of multiple positive solutions of nonlinear boundary value problems of local and nonlocal type, Topol. Methods Nonlinear Anal., 27(2006), 91-116.

[20] Q. Yao, Positive solution for a semi-linear third-order two-point boundary value problem, Appl. Math. Lett., 17(2004), 1171-1175.

Received: November 28, 2018; Accepted: March 6, 2021. 